\title{
Modeling the Power Angular Profile of Dense Multipath Components Using Multiple Clusters
}

\author{
Brecht Hanssens ${ }^{1}$, Kentaro Saito ${ }^{2}$, Emmeric Tanghe ${ }^{1}$, Luc Martens ${ }^{1}$, \\ Claude Oestges $^{3}$, Wout Joseph ${ }^{1}$, Jun-ichi Takada ${ }^{2}$ \\ ${ }^{1}$ INTEC-WAVES, Ghent University / imec, Ghent, Belgium, Brecht.Hanssens@UGent.be \\ ${ }^{2}$ School of Environment and Society, Tokyo Institute of Technology, Tokyo, Japan. \\ ${ }^{3}$ ICTEAM, Université catholique de Louvain, Louvain-la-Neuve, Belgium.
}

\begin{abstract}
In this work, we will discuss the modeling of the Power Angular Profile (PAP) of the Dense Multipath Components (DMC), taking into account multiple angular clusters. We have extended the maximum likelihood estimation of the angular DMC parameters in the RiMAX framework, so that the PAP of the DMC can be modeled with a multimodal von Mises distribution. This allows us to better characterize the diffuse scattering between transmitter and receiver, originating from multiple reflections in an environment. We have validated our proposed method with synthetic radio channel data based on Monte Carlo simulations, and have shown the importance of applying a multimodal assumption over a unimodal one.
\end{abstract}

Index Terms-Channel Modeling, Multipath Estimation, RiMAX, Angular Modeling, Polarization, DMC

\section{INTRODUCTION}

Over the recent years, mobile networks are becoming heavily congested due to the continuous increase in user traffic, caused by the emergence of wireless systems, mobile applications and streaming services. To accommodate for this additional demand in wireless signal spectrum, nextgeneration mobile communication systems such as $5 \mathrm{G}$ are currently being investigated. In the frequency bands utilized by $5 \mathrm{G}$, massive Multiple-Input Multiple-Output (MIMO) technologies are expected to be employed to further enhance the data transmission capabilities of these systems, by relying on higher order beamforming and spatial multiplexing. The primary spectrum that is considered for the lower $5 \mathrm{G}$ bands all range between $1 \mathrm{GHz}$ and $6 \mathrm{GHz}$, of which it is well known that their channel capacities are highly influenced by the amount of diffuse scattering present in the radio channel. Diffuse scattering arises from radio waves scattering on electrically small and rough surfaces, which are inherently more present at sub-20 GHz frequencies [1], in contrast to the higher $5 \mathrm{G}$ frequency bands (between $24 \mathrm{GHz}$ and $30 \mathrm{GHz}$ ), mainly due to their shorter wavelengths and less attenuation. In indoor environments, where radio waves typically scatter from various objects, diffuse scattering plays an even more important role in the MIMO transmission capacity [2]. However, its contribution is not taken into account in the standard channel models such as the 3GPP Spatial Channel Model (SCM) [3], the ITU-R M.2135 channel model [4], or the WINNER II channel model [5]. The novelty is that we consider the existence of multiple angular clusters in the residual power spectrum of the radio channel, allowing us to construct a model that characterizes multiple reflections of the electromagnetic waves at different angles in the environment.

The structure of this paper is as follows. Section II summarizes the related work on this topic, whilst Section III describes the applied channel model with the angular modeling of the diffuse scattering. Section IV presents our simulation setup and the resulting outcome. Finally, Section V summarizes this paper with a conclusion and ideas for future work.

\section{RELATED WORK}

Recent works such as [6], [7] estimated the contributions of diffuse scattering directly from MIMO radio channel measurements by applying the RiMAX multipath estimation framework to estimate both the Specular and the Dense Multipath Components (SMC and DMC) from the measurement data. The SMC can be seen as the deterministic part of the radio channel, comprised of a number of plane waves with well defined propagation parameters in multipath space (space, frequency, and time-delay domain). They concern the coherent waves in the channel, which can either be direct waves from transmitter to receiver, or specular reflections due to mirror-like interactions with the environment. The DMC can be seen as the stochastic part of the radio channel, which is continuous in the aforementioned dimensions. They originate mainly from diffuse scattering on electrically small and rough surfaces, and also contain the unresolvable SMC contributions which can not be estimated accurately enough with a multipath estimation framework. The contributions of DMC are characterized by autocorrelations of the signal component, but the RiMAX framework [6], [7] only discusses the frequency domain correlation modeling of the DMC.

Investigations into the behavior of the angular DMC parameters found that correlations exist between the locations of the SMC and the dominating powers in the DMC spectrum. [8] and [9] proposed to model the DMC profile as clusters around the SMC. In [10], [11], a maximum-likelihood (ML) method is utilized based on a unimodal von Mises distribution to model the angular profile of the DMC. In [12], this distribution was also found to be a good fit to model the angular domain autocorrelation of the DMC in the $11 \mathrm{GHz}$ band, by convolution of the DMC angular profile with the antenna array response. 
In this work, we will extend the ML method in the RiMAX algorithm from a unimodal to a multimodal assumption. As such, we are able to model multiple angular clusters in the DMC spectrum of the radio channel, originating from multiple reflections of the plane waves in an environment. As was shown in [12] and [13], the DMC increases the level of the reconstructed eigenvalues, resulting in a better approximation of the measured eigenvalue structure of the MIMO channel. This indicates that an accurate modeling of the DMC parameters is necessary to prevent the underestimation of the MIMO transmission performance [2]. This paper extends our previous work on this topic [12], [14] by extending the conventional RiMAX framework for the joint estimation of multimodal DMC propagation parameters in the frequency, angular and polarization domains. We have validated our proposed method with synthetic radio channel data based on extensive Monte Carlo simulations.

\section{Channel Model}

An observation of the MIMO radio channel $h$ can be modeled as the superposition of Specular Multipath Components (SMC), and Dense Multipath Components (DMC), and can be written as follows:

$$
\boldsymbol{h}=\boldsymbol{s}\left(\boldsymbol{\theta}_{s}\right)+\boldsymbol{d}\left(\boldsymbol{\theta}_{d}\right),
$$

in which $\boldsymbol{h}$ can be seen as a random variable distributed according to a complex multivariate Gaussian distribution:

$$
\boldsymbol{h} \sim \mathcal{N}_{c}\left(\boldsymbol{s}\left(\boldsymbol{\theta}_{s}\right), \boldsymbol{R}\left(\boldsymbol{\theta}_{d}\right)\right) .
$$

This work only concerns the DMC part of the channel, so we refer to [15] for a discussion of the modeling of the SMC.

\section{A. Unimodal assumption of DMC in angular domain}

The DMC is modeled stochastically by means of the covariance matrix of the residual (dense) part of the radio channel $\boldsymbol{R}\left(\boldsymbol{\theta}_{d}\right)$, based on the DMC propagation parameters $\boldsymbol{\theta}_{d, F}$ in the time-delay domain, and $\boldsymbol{\theta}_{d, A}$ in the angularpolarization domain, defined as follows:

$$
\begin{aligned}
\boldsymbol{\theta}_{d, F} & =\left[\alpha_{0}, \alpha_{1}, \beta_{d}, \tau_{d}\right] \\
\boldsymbol{\theta}_{d, A} & =\left[\mu_{T}, \mu_{R}, \kappa_{T}, \kappa_{R}, \gamma_{d, v v}, \gamma_{d, v h}, \gamma_{d, h v}, \gamma_{d, h h}, \gamma_{\alpha}\right] .
\end{aligned}
$$

In [10], [16], the Power Angular Profile (PAP) of the DMC at the transmitter (Tx) or the receiver $(\mathrm{Rx})$ in the azimuth plane is modeled using a unimodal von Mises distribution, which is defined (e.g., at Tx) as follows:

$$
f_{T}(\varphi)=\frac{1}{2 \pi \mathrm{I}_{0}\left(\kappa_{T}\right)} \exp \left(\kappa_{T} \cos \left(\varphi-\mu_{T}\right)\right),
$$

with $\mathrm{I}_{0}\left(\kappa_{T}\right)$ being the modified Bessel function of the first kind and zeroth order, the parameter $\mu_{T}$ controlling the center location of the distribution at $\mathrm{Tx}$, and $\kappa_{T}$ controlling its angular spread. As the value for $\kappa_{T}$ increases, the angular spread of the distribution decreases, and the von Mises distribution approaches the normal distribution. For a $\kappa_{T}$ value of 0 , the von Mises distribution becomes the uniform distribution.
In the polarimetric scenario, the PAP is modeled by multiplying the von Mises distribution with an angle-independent polarization vector $\gamma_{d}=\left[\gamma_{d, v v}, \gamma_{d, v h}, \gamma_{d, h v}, \gamma_{d, h h}, \gamma_{\alpha}\right]$ [11]. The angular and polarization domain covariance matrix $\boldsymbol{R}_{A}\left(\boldsymbol{\theta}_{d, A}\right) \in \mathbb{C}^{N_{T} N_{R} \times N_{T} N_{R}}$ can then be written as follows:

$$
\boldsymbol{R}_{A}\left(\boldsymbol{\theta}_{d, A}\right)=\boldsymbol{R}_{v v}+\boldsymbol{R}_{v h}+\boldsymbol{R}_{h v}+\boldsymbol{R}_{h h}+\gamma_{\alpha} \boldsymbol{I} .
$$

In Eq. (6), the angular domain covariance matrix $\boldsymbol{R}_{x y}$ for a certain polarization setting $x y$ (e.g., Tx-V and $\mathrm{Rx}-\mathrm{H}$; denoted as $\mathrm{VH}$ ) can be written as:

$$
\boldsymbol{R}_{x y}=\gamma_{d, x y} \boldsymbol{C}_{x y},
$$

in which $C_{x y}$ is the combined Tx and Rx angular power spectrum, defined as the Kronecker-product between two matrices $C_{x}$ and $C_{y}$, representing their separate angular power spectrum. We can thus write the following:

$$
\begin{aligned}
\boldsymbol{C}_{x y} & =\boldsymbol{C}_{x} \otimes \boldsymbol{C}_{y} \\
\boldsymbol{C}_{x} & =\int_{-\pi}^{\pi} \boldsymbol{\alpha}_{T, x}(\varphi) f_{T}(\varphi) \boldsymbol{\alpha}_{T, x}^{H}(\varphi) d \varphi \\
\boldsymbol{C}_{y} & =\int_{-\pi}^{\pi} \boldsymbol{\alpha}_{R, y}(\varphi) f_{R}(\varphi) \boldsymbol{\alpha}_{R, y}^{H}(\varphi) d \varphi .
\end{aligned}
$$

In (9) and (10), $\boldsymbol{\alpha}_{T, x}$ and $\boldsymbol{\alpha}_{R, y}$ are the antenna array responses of the transmitter for the polarization setting $x$, and the receiver for the polarization setting $y$, respectively. Finally, the full DMC correlation matrix $\boldsymbol{R}$ is calculated as follows:

$$
\boldsymbol{R}=\boldsymbol{R}_{F}\left(\boldsymbol{\theta}_{d, F}\right) \otimes \boldsymbol{R}_{A}\left(\boldsymbol{\theta}_{d, A}\right) .
$$

In this work, we will only focus on the estimation of the angular DMC parameters, so that we will model the correlation matrix in the time-delay domain $\boldsymbol{R}_{F}\left(\boldsymbol{\theta}_{d, F}\right)$ as a unitary matrix.

\section{B. Multimodal assumption of DMC in angular domain}

In the multimodal assumption of the angular DMC power spectrum, multiple clusters exists in the PAP of Tx and Rx, each with their own von Mises distribution defined by a center location $\mu$ and an angular spread $\kappa$. As such, we can re-write $\boldsymbol{R}_{x y}$ as being the summation over several separate covariance matrices, which can be written as follows:

$$
\boldsymbol{R}_{x y}=\sum_{c_{t}=1}^{C_{T}} \sum_{c_{r}=1}^{C_{R}} \gamma_{d, x y}^{c_{t}, c_{r}} \boldsymbol{C}_{x y}^{c_{t}, c_{r}},
$$

with $C_{T}$ and $C_{R}$ the number of clusters in the PAP of Tx and Rx, respectively. It follows that $C_{x y}^{c_{t}, c_{r}}$ then becomes the Kronecker-product between both $\boldsymbol{C}_{x}^{c_{t}}$ and $\boldsymbol{C}_{y}^{c_{r}}$ as follows:

$$
\begin{aligned}
\boldsymbol{C}_{x y}^{c_{t}, c_{r}} & =\boldsymbol{C}_{x}^{c_{t}} \otimes \boldsymbol{C}_{y}^{c_{r}} \\
\boldsymbol{C}_{x}^{c_{t}} & =\int \boldsymbol{\alpha}_{T, x}(\varphi) f_{T}^{c_{t}}(\varphi) \boldsymbol{\alpha}_{T, x}^{H}(\varphi) d \varphi \\
\boldsymbol{C}_{y}^{c_{r}} & =\int \boldsymbol{\alpha}_{R, y}(\varphi) f_{R}^{c_{r}}(\varphi) \boldsymbol{\alpha}_{R, y}^{H}(\varphi) d \varphi,
\end{aligned}
$$

in which $f_{T}^{c_{t}}(\varphi)$ and $f_{R}^{c_{r}}(\varphi)$ are both unimodal von Mises distributions of the $c_{t}$-th cluster at Tx, and the $c_{r}$-th cluster 
at $\mathrm{Rx}$, respectively. As such, the combined PAP at Tx and $\mathrm{Rx}$ will consist of a multitude of angular clusters, so that the DMC angular parameters of multiple reflections at different angles in the environment can be better characterized.

It should be noted that the total angular DMC parameter set $\boldsymbol{K}$ for either a unimodal or a multimodal assumption is equal to the following:

$$
\boldsymbol{K}=\left[\left(C_{T}+C_{R}\right)\{\mu, \kappa\}, C_{T} C_{R}\left\{\gamma_{v v}, \gamma_{v h}, \gamma_{h v}, \gamma_{h h}\right\}, \gamma_{\alpha}\right],
$$

such that the number of parameters $L$ to be estimated is equal to $|\boldsymbol{K}|=1+2\left(C_{T}+C_{R}\right)+4\left(C_{T}+C_{R}\right)$. A single cluster at both $\mathrm{Tx}$ and $\mathrm{Rx}$ (unimodal assumption) leads to the estimation of 9 angular DMC parameters, whilst 2 clusters at both Tx and Rx already makes 25 , and 3 clusters even makes 49. This will no doubt have its consequences regarding the accuracy of the estimation procedure.

\section{Estimator for the multimodal angular DMC model}

The log-likelihood function of RiMAX can be calculated by using the correlation matrix $\tilde{\boldsymbol{R}}$ of the residual DMC spectrum $\boldsymbol{d}\left(\boldsymbol{\theta}_{d}\right)$, which is defined in the angular-polarization domain as follows:

$$
\begin{aligned}
\mathcal{L}_{A}\left(\boldsymbol{h} \mid \boldsymbol{\theta}_{d, A}\right)=-\ln \left(\operatorname{det}\left(\boldsymbol{R}_{A}\left(\boldsymbol{\theta}_{d, A}\right)\right)\right) & \\
- & \operatorname{tr}\left(\boldsymbol{R}_{A}\left(\boldsymbol{\theta}_{d, A}\right)^{-1} \tilde{\boldsymbol{R}}_{A}\right)+C,
\end{aligned}
$$

in which $C$ is a constant. We can then use the initialization and the optimization procedure for the angular DMC parameters as described in [11], by extending its linear model from a unimodal to a multimodal angular DMC assumption, as described below. First, we need to write the total angleindependent polarimetric power vector $\Gamma_{d}^{c_{t}, c_{r}}$, representing the polarimetric power between the angular DMC clusters $c_{t}$ at $\mathrm{Tx}$, and $c_{r}$ at $\mathrm{Rx}$, as follows:

$$
\Gamma_{d}^{c_{t}, c_{r}}=\operatorname{vec}\left\{\left[\gamma_{d, v v}^{c_{t}, c_{r}}, \gamma_{d, v h}^{c_{t}, c_{r}}, \gamma_{d, h v}^{c_{t}, c_{r}}, \gamma_{d, h h}^{c_{t}, c_{r}}\right]\right\}
$$

in which $\operatorname{vec}\{\cdot\}$ is an operator that reshapes a matrix into a row vector. We can then construct a model from Eq. (6), relying on the linear dependences of the covariance matrix $\boldsymbol{R}_{A}$ as follows:

$$
\begin{aligned}
\boldsymbol{R}_{A, v}= & \operatorname{vec}\left\{\boldsymbol{R}_{A}\right\}^{T} \\
\boldsymbol{R}_{A, v}= & \sum_{c_{t}=1}^{C_{T}} \sum_{c_{r}=1}^{C_{R}} \boldsymbol{\Gamma}_{d}^{c_{t}, c_{r}} \boldsymbol{C}_{v}^{c_{t}, c_{r}}+\gamma_{\alpha} \boldsymbol{I} \\
\boldsymbol{C}_{v}^{c_{t}, c_{r}}= & {\left[\begin{array}{l}
\operatorname{vec}\left\{\boldsymbol{C}_{v v}^{c_{t}, c_{r}}\right\}^{T} \\
\operatorname{vec}\left\{\boldsymbol{C}_{v h}^{c_{t}, c_{r}}\right\}^{T} \\
\operatorname{vec}\left\{\boldsymbol{C}_{h v}^{c_{t}, c_{r}}\right\}^{T} \\
\operatorname{vec}\left\{\boldsymbol{C}_{h h}^{c_{t}, c_{r}}\right\}^{T}
\end{array}\right], }
\end{aligned}
$$

This linear model can then be used to find an initial set of multimodal angular DMC parameters by using the beamforming based method as described in [11]. The angular-polarization domain DMC propagation parameter $\boldsymbol{\theta}_{d, A}$ can then be optimized by making use of the NewtonRaphson algorithm. Firstly, the score function $\boldsymbol{q}_{A}\left(\boldsymbol{h} \mid \boldsymbol{\theta}_{d, A}\right)$ and the Fisher information matrix $\boldsymbol{J}_{A}\left(\boldsymbol{\theta}_{d, A}\right)$ of the angularpolarization domain log-likelihood function $\mathcal{L}_{A}\left(\boldsymbol{h} \mid \boldsymbol{\theta}_{d, A}\right)$ need to be calculated as follows:

$$
\begin{array}{r}
\boldsymbol{q}_{A}\left(\boldsymbol{h} \mid \boldsymbol{\theta}_{d, A}\right)=\operatorname{tr}\left(\boldsymbol{R}_{A}\left(\boldsymbol{\theta}_{d, A}^{(i t r)}\right)^{-1}\left[\frac{\partial \boldsymbol{R}_{A}\left(\boldsymbol{\theta}_{d, A}^{(i t r)}\right)}{\partial \boldsymbol{\theta}_{d, A}}\right]\right. \\
\left.\boldsymbol{R}_{A}\left(\boldsymbol{\theta}_{d, A}^{(i t r)}\right)^{-1} \tilde{\boldsymbol{R}}_{A}-\boldsymbol{I}\right) \\
\boldsymbol{J}_{A}\left(\boldsymbol{\theta}_{d, A}\right)_{i j}=\operatorname{tr}\left(\boldsymbol{R}_{A}\left(\boldsymbol{\theta}_{d, A}^{(i t r)}\right)^{-1} \frac{\partial \boldsymbol{R}_{A}\left(\boldsymbol{\theta}_{d, A}^{(i t r)}\right)}{\partial \theta_{d, A, j}}\right. \\
\left.\boldsymbol{R}_{A}\left(\boldsymbol{\theta}_{d, A}^{(i t r)}\right)^{-1} \frac{\partial \boldsymbol{R}_{A}\left(\boldsymbol{\theta}_{d, A}^{(i t r)}\right)}{\partial \theta_{d, A, i}}\right) .
\end{array}
$$

The propagation parameter $\boldsymbol{\theta}_{d, A}^{(i t r)}$ is then updated by the incremental step $\Delta \boldsymbol{\theta}_{d, A}^{(i t r)}$ as follows:

$$
\begin{aligned}
& \Delta \boldsymbol{\theta}_{d, A}^{(i t r)}=-\boldsymbol{J}_{A}\left(\boldsymbol{\theta}_{d, A}^{(i t r)}\right)^{-1} \boldsymbol{q}_{A}\left(\boldsymbol{h} \mid \boldsymbol{\theta}_{d, A}^{(i t r)}\right) \\
& \boldsymbol{\theta}_{d, A}^{(i t r+1)}=\boldsymbol{\theta}_{d, A}^{(i t r)}+\lambda_{A} \Delta \boldsymbol{\theta}_{d, A}^{(i t r)},
\end{aligned}
$$

in which $\lambda_{A}$ is the step length, initialized at 1 . If the resulting updated parameter $\boldsymbol{\theta}_{d, A}^{(i t r+1)}$ causes an increase in the loglikelihood function Eq. (17), convergence is achieved, and we will start the next iteration with the optimization of the time-delay DMC propagation parameter. If not, $\lambda_{A}$ is divided by 4 , and the algorithm keeps searching for a new updated parameter $\boldsymbol{\theta}_{d, A}^{(i t r+1)}$ until convergence is achieved.

\section{Simulations}

\section{A. Simulation setup}

In total, 1000 Monte Carlo simulations were performed by sampling random values per simulation run for the angular DMC parameters within a certain range, and running the initialization and optimization procedures as summarized above. In this work, we have assumed a fixed value of two clusters at both $\mathrm{Tx}$ and $\mathrm{Rx}$, respectively. When processing real measurement data, a technique should be devised in order to estimate how many clusters exist in the residual DMC spectrum at both $\mathrm{Tx}$ and $\mathrm{Rx}$, respectively.

The generated center values $\mu$ of our von Mises distributions were randomly sampled from the interval $\left[0^{\circ}: 360^{\circ}\right.$, with the additional condition that the center value of the second cluster needs to be at least $50^{\circ}$ away from the first cluster. This ensures that a proper matching can be performed between the generated and the estimated clusters before calculating their estimation errors, since their center values can be distinguished enough during the evaluation phase. The angular spread of each cluster, regulated by the parameter $\kappa$, was randomly sampled from the interval $[0.5: 5]$. This corresponds with a standard deviation of $96.45^{\circ}$ and $27.21^{\circ}$, respectively. The angle-independent polarization matrix $\gamma_{d}$ was sampled randomly between $[0.2: 1]$ for each cluster combination $c_{t}, c_{r}$, and for each polarization setting $x y$, and the noise power $\gamma_{\alpha}$ was sampled randomly between [0.05: 0.15]. 


\section{B. Unimodal modeling of a multimodal spectrum}

The main purpose of this work is to be able to characterize multiple peaks in the power level in the residual angular DMC spectrum at both Tx and Rx, at those angles of departure and arrival contributing to diffuse reflections of the electromagnetic waves in the environment. Figure 1 shows a generated multimodal spectrum containing two clusters (resulting in two peaks), which we processed first by using a unimodal assumption, and then by using a multimodal assumption.

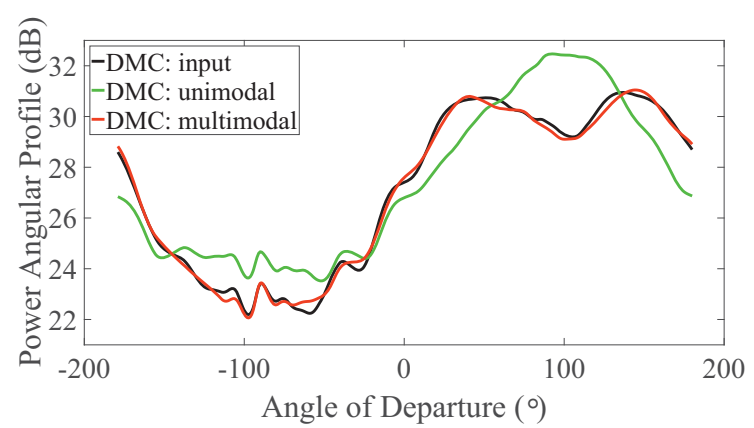

(a) Transmitter

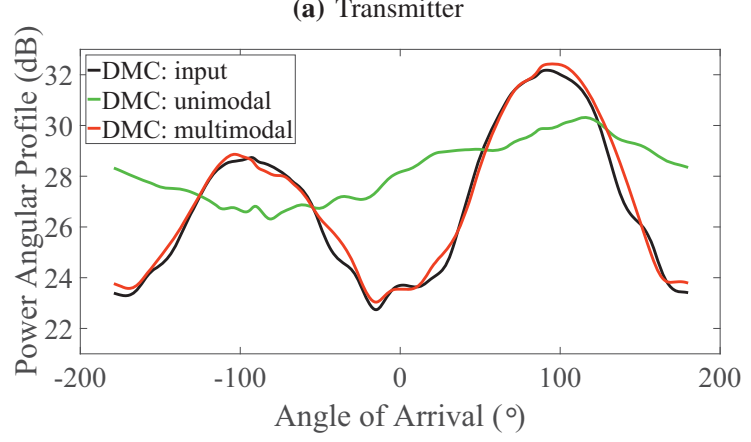

(b) Receiver

Figure 1: Power Angular Profile of the DMC. The black line denotes the generated input DMC spectrum, red is the estimated multimodal DMC spectrum, and green is the estimated unimodal DMC spectrum.

From Figure 1, we can observe that if we would model an angular DMC spectrum with multiple clusters by using a unimodal assumption (i.e., with a unimodal distribution), both the center values and the angular spread of this distribution can be wrongfully estimated. When looking at the two input clusters in Figure 1a, the multimodal distribution fits these very well, whilst the unimodal distribution estimates the center value somewhere in between them. When looking at Figure $1 \mathrm{~b}$, the unimodal distribution tends to estimate its center value more towards the stronger cluster, whilst its estimate for the angular spread pushes the distribution such that it tries to average out the entire DMC spectrum. However, if we model this spectrum using a multimodal assumption, both generated center values are estimated correctly, and their angular spreads correspond very well with the true generated values.

\section{Simulation results}

Table I shows the estimation errors between the generatedand the estimated von Mises distributions (i.e., clusters) at Tx and $\mathrm{Rx}$. The $\mu$ error denotes the difference between the generated and the estimated center value of both distributions. The $\kappa$ error denotes the difference in angular spread (converted into degrees), whilst the $\gamma_{d}$ error denotes the difference in relative power (summed over all polarizations) between each generated and estimated cluster (expressed in \%).

\begin{tabular}{|c|c|cc|cc|cc|}
\hline \multicolumn{2}{|c|}{} & \multicolumn{2}{|c|}{$\mu$ error $\left(^{\circ}\right)$} & \multicolumn{2}{c|}{$\kappa$ error $\left(^{\circ}\right)$} & \multicolumn{2}{c|}{$\gamma_{d}$ error $(\%)$} \\
\cline { 3 - 8 } \multicolumn{2}{|c|}{$\mathbf{T x}$} & $\# 1$ & $\# 2$ & $\# 1$ & $\# 2$ & $\# 1$ & $\# 2$ \\
\hline \multirow{2}{*}{$\mathbf{R x}$} & mean & 0.04 & -0.13 & -0.06 & -0.05 & -0.09 & -0.08 \\
& stdvar & 3.12 & 2.56 & 1.95 & 2.67 & 3.24 & 3.22 \\
& mean & 0.14 & 0.08 & -0.03 & -0.05 & -0.18 & -0.10 \\
& stdvar & 4.62 & 5.73 & 2.42 & 2.68 & 4.00 & 4.28 \\
\hline
\end{tabular}

Table I: Estimation errors between the generated- and the estimated von Mises distributions (i.e., clusters) at Tx and Rx. Distribution \#1 always denotes the stronger cluster, whilst distribution \#2 denotes the weaker cluster.

From Table I, we can conclude that our algorithm is able to estimate the generated clusters with a mean error of less than $1^{\circ}$ for both the center values and the angular spreads of each cluster. The mean error in relative power is limited to less than one tenth of a percent. Our algorithm achieved convergence in less than 25 iterations, with a mean number of 5.86 iterations.

Figures 2, 3 and 4 show the estimation errors between the generated and the estimated DMC parameters of the von Mises distributions as a histogram, of both the strongest (red) and the weakest (blue) cluster. The color purple indicates the overlap between both (red and blue) histograms.

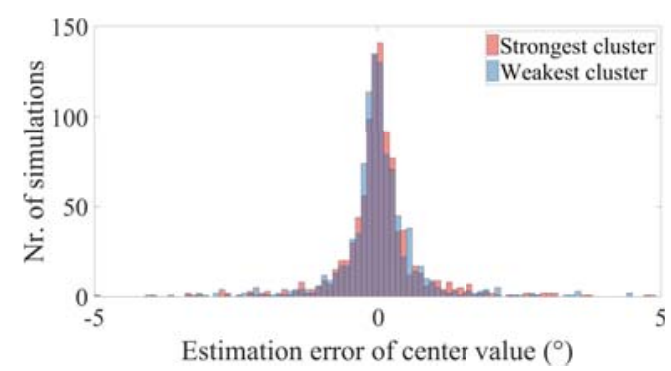

(a) Transmitter

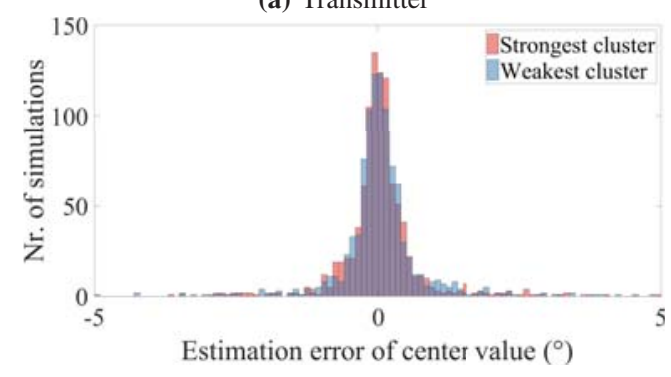

(b) Receiver

Figure 2: Histogram of the estimation errors between the generated- and the estimated center values of each cluster.

\section{Conclusions}

In this work, we have extended the maximum likelihood estimation of angular DMC parameters in the RiMAX algorithm from a unimodal to a multimodal assumption, in 


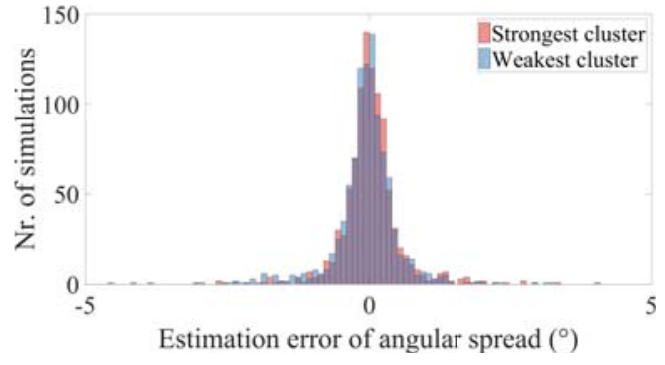

(a) Transmitter

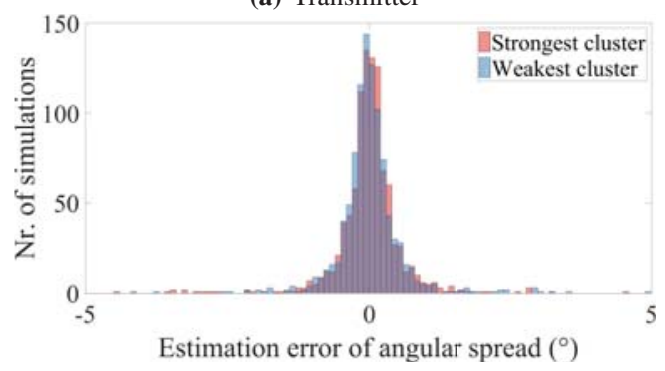

(b) Receiver

Figure 3: Histogram of the estimation errors between the generated- and the estimated angular spreads (converted into degrees) of each cluster.

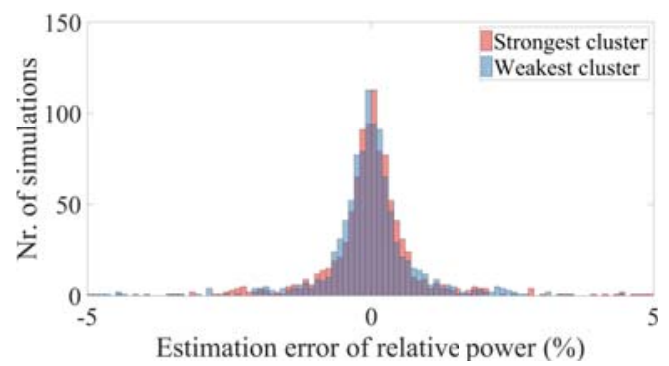

(a) Transmitter

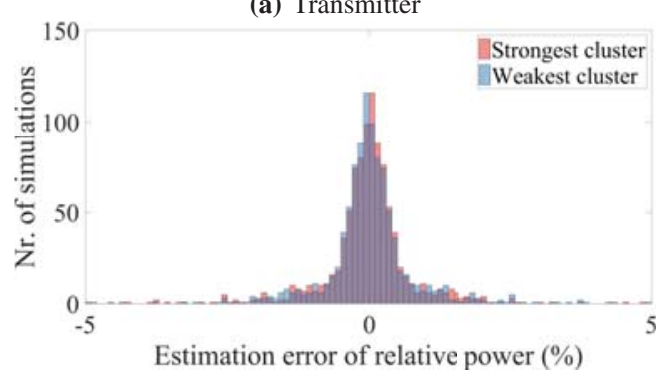

(b) Receiver

Figure 4: Histogram of the estimation errors between the generated- and the estimated relative powers (expressed in \%) of each cluster.

order to account for multiple angular clusters in the residual DMC spectrum. Monte Carlo simulation showed that our algorithm is able to estimate the generated clusters with an accuracy of less than $1^{\circ}$ for both the center values and the angular spreads of each cluster. Modeling the angular DMC spectrum by applying a unimodal assumption can sometimes be erroneous, resulting in the fact that both the center values and the angular spreads in this spectrum can be wrongfully estimated. A correct modeling of the DMC parameters is thus necessary to accurately characterize the true MIMO transmission performance, highlighting the importance of taking into account a multimodal assumption over a unimodal one.

\section{ACKNOWLEDGMENT}

Brecht Hanssens is funded by a Ph.D. grant of the Agency for Innovation by Science and Technology (IWT). Emmeric Tanghe is a Post-Doctoral Fellow of the Research Foundation Flanders (FWO). This work was carried out in the framework of COST Action CA15104 IRACON, and was supported by the FWO project G027714N, IAP BESTCOM, "The research and development for expansion of radio spectrum resources" of The Ministry of Internal Affairs and Communications, and JSPS KAKENHI Grants Numbers 15H04003 and 16K18102.

\section{REFERENCES}

[1] A. Bamba, M. T. Martinez-Ingles, D. P. Gaillot, et al. Experimental Investigation of Electromagnetic Reverberation Characteristics as a Function of UWB Frequencies. IEEE Antennas and Wireless Propagation Letters, 14:859-862, 2015.

[2] A. Richter, J. Salmi, and V. Koivunen. Distributed scattering in radio channels and its contribution to MIMO channel capacity. In European Conference on Antennas and Propagation (EuCAP), pages 1-7, 2006.

[3] 3GPP. Spatial channel model for Multiple Input Multiple Output (MIMO) simulations (Release 7), 3GPP TR 25. 996, v7. 0. 0, 2007.

[4] ITU-R. Guidelines for evaluation of radio interface technologies for IMT-Advanced, Report ITU-R M.2135, 2009. [Online]. Available: http://www.itu.int/pub/R-REP-M.2135-2008/en.

[5] P. Kyösti, J. Meinilä, L. Hentilä, et al. WINNER II Channel Models. 2007.

[6] A. Richter. Estimation of Radio Channel Parameters: Models and Algorithms. PhD thesis, Technische Universität Ilmenau, Fakultät für Elektrotechnik und Informationstechnik, Ilmenau, Germany, 2005.

[7] A. Richter and R. Thoma. Joint maximum likelihood estimation of specular paths and distributed diffuse scattering. In IEEE conference on Vehicular Technology Conference (VTC), volume 1, pages 11-15, 2005.

[8] J. Poutanen, J. Salmi, K. Haneda, et al. Propagation Characteristics of Dense Multipath Components. IEEE Antennas and Wireless Propagation Letters, 9:791-794, 2010.

[9] F. Quitin, C. Oestges, F. Horlin, et al. Diffuse Multipath Component Characterization for Indoor MIMO Channels. In European Conference on Antennas and Propagation (EUCAP), pages 1-5, 2010.

[10] C. B. Ribeiro, A. Richter, and V. Koivunen. Joint Angular- and DelayDomain MIMO Propagation Parameter Estimation Using Approximate ML Method. IEEE Transactions on Signal Processing (TAP), 55(10):4775-4790, 2007.

[11] M. Kaske and R. Thoma. Maximum-Likelihood based estimation of angular parameters of Dense-Multipath-Components. In European Conference on Antennas and Propagation (EuCAP), pages 1-6, 2015.

[12] K. Saito, J. i. Takada, and M. Kim. Dense Multipath Component Characteristics in $11 \mathrm{GHz}$-band Indoor Environments. IEEE Transactions on Antennas and Propagation, PP(99):1-1, 2017.

[13] U. T. Virk, K. Haneda, and J. F. Wagen. Dense Multipath Components Add-on for COST 2100 Channel Model. In 2015 9th European Conference on Antennas and Propagation (EuCAP), pages 1-5, 2015.

[14] K. Saito, J. i. Takada, and M. Kim. Dense Multipath Component Parameter Estimation in 11GHz-band Indoor Environment. In 2016 IEEE 27th Annual International Symposium on Personal, Indoor, and Mobile Radio Communications (PIMRC), pages 1-6, 2016.

[15] J. A. Fessler and A. O. Hero. Space-Alternating Generalized Expectation-Maximization Algorithm. IEEE Transactions on Signal Processing, 42(10):2664-2677, 1994.

[16] J. Salmi, J. Poutanen, K. Haneda, et al. Incorporating diffuse scattering in geometry-based stochastic MIMO channel models. In European Conference on Antennas and Propagation (EUCAP), pages 1-5, 2010. 